\title{
Comparison and Applicability Analysis of Dynamics Models for Piezoelectric Energy Harvester
}

\author{
Bing Chen, Yu-tong Shi, Li-jie Zhang \\ School of Mechanical Engineering, University of Science and Technology Beijing \\ 30 Xueyuan Road, Haidian District, Beijing, 100083 P. R.China \\ bingchen9803@ustb.edu.cn; 646133782@qq.com
}

\begin{abstract}
The dynamic model of piezoelectric energy harvester mainly includes two types: continuum model and lumping model. However, when selecting the above two types of modelling algorithms, the accuracy and calculation background requirements related to the indicators such as resonance frequency and peak value are rarely considered. Therefore, the two types of models can be used to analyse the same piezoelectric cantilever beam oscillator, and the experimental data are used for comparative study. The results show that the results obtained by the two algorithm s can accurately output the voltage change trend. The lumping model is simple but not precise, and the continuum model approach is relatively complex but more accurate.
\end{abstract}

Keywords: Energy harvester, Cantilever beam, Continuum, Lumping.

\section{Introduction}

The piezoelectric vibration energy capture system needs to be theoretically analyzed [1]. In order to describe and analyze the electromechanical coupling behavior of linear piezoelectric generators, scholars at home and abroad have carried out a lot of research and proposed many modeling algorithms. Generally speaking, the theoretical modeling of piezoelectric cantilever vibration energy harvester is mainly based on two types of models: lumping model [2][3] and continuum model [4][5]. The lumping model is often used in simple theoretical modeling and engineering application research because of its simplicity and intuitiveness. The continuum model uses the Euler-Bernoulli equation and it uses the Euler-Bernoulli equation to consider the force of each point of the cantilever beam on the basis of the finite element theory [6].

In this paper, the traditional piezoelectric cantilever beam structure is improved, and a new multimode piezoelectric vibration energy harvester is proposed. The system consists of PZT cantilever beam and resonator, and then the system of motion control equation, frequency characteristic equation and electric output power equation model are established by lumping model and continuum model respectively. Finally, the characteristics of the above two models are verified by the comparison of simulation data and experimental data, and their respective scopes are explored.

\section{Multimodal vibration energy harvester model}

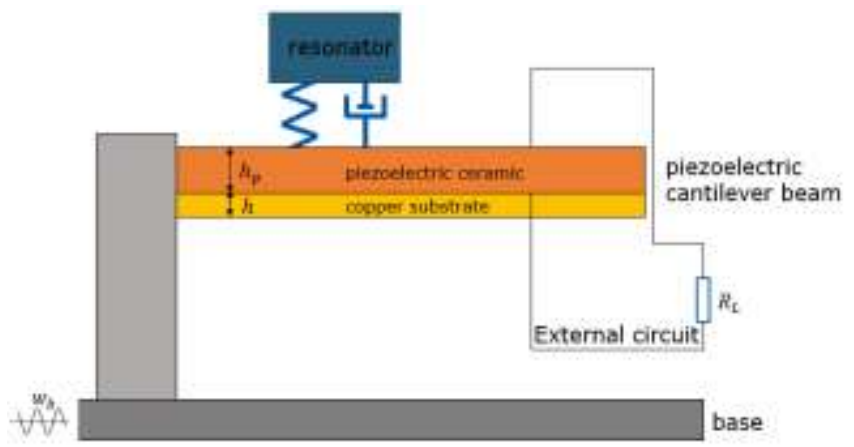

Fig. 1: Schematic diagram of a new type of piezoelectric cantilever vibration energy harvester. 
Conventional piezoelectric cantilever structures are difficult to match multiple resonant frequencies with excitation frequencies, and multimode energy harvesting can't be achieved [7]. Therefore, adding resonator components, and forming a spring-mass block vibration subsystem, the operating frequency of the energy harvester can be effectively matched with the excitation frequency to achieve multimodal collection of vibration energy. The piezoelectric vibration energy capture system needs to be theoretically analyzed. In order to describe and analyze the electromechanical coupling behavior of linear piezoelectric generators, scholars at home and abroad have carried out a lot of research and proposed many modeling algorithms. Generally speaking, the theoretical modeling of piezoelectric cantilever vibration energy harvester is mainly based on two types of models: lumping model and continuum model. The lumping model is often used in simple theoretical modeling and engineering application research because of its simplicity and intuitiveness. The continuum model uses the Euler-Bernoulli equation and it uses the Euler-Bernoulli equation to consider the force of each point of the cantilever beam on the basis of the finite element theory.

\section{Mathematical Model Analysis of Piezoelectric Cantilever Beam Vibration Energy Harvester 3.1. Lumping model and analysis}

As shown in Fig.2, the original piezoelectric energy harvester is equivalent to a two-degree-of-freedom dynamic model, with an ideal circuit model attached. $k_{a}$ is the equivalent stiffness, $m_{b}$ is the equivalent mass, $c_{b}$ is the equivalent damping, $w_{b}$ is the base displacement, $z_{l}$ is the absolute displacement of the equivalent mass $m_{b}, z_{b}=z_{l}-w_{b}$ is the relative displacement of the equivalent mass and the pedestal, $m_{a}$ is the resonator mass, $k_{a}$ and $c_{a}$ are the spring stiffness and damping coefficient of the resonator, respectively; $z_{a}$ is the displacement of the resonator relative to the cantilever beam.

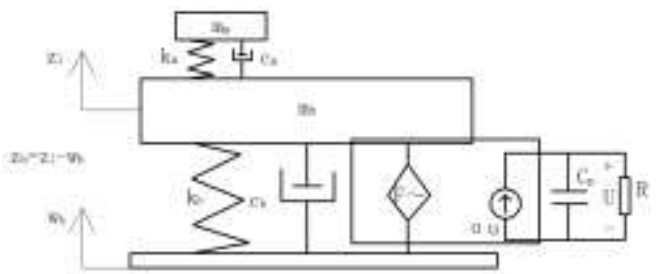

Fig. 2: Electromechanical coupling model of system lumping model.

According to the principle of mechanical and electrical balance, the equation of motion of the piezoelectric cantilever vibration energy capture system is [8]:

$$
m_{b} \ddot{z}_{b}(t)+c_{b} \dot{z}_{b}(t)+k_{b} z_{b}(t)+\alpha u(t)-\left(c_{a} \dot{z}_{a}(t)+k_{a} z_{a}(t)\right)=-m_{b} \ddot{w}_{b}(t)
$$

where $u(t)$ is the output voltage of the piezoelectric generator, which is the voltage across the load $R_{L}, \alpha$ is the electromechanical coupling constant of the piezoelectric generator, and $\alpha u(t)$ is the force generated by the piezoelectric effect of the cantilever beam piezoelectric vibration harvester, $\ddot{w}_{b}(t)$ is the acceleration form of the displacement excitation.

According to the principle of mechanical balance, the equation of motion of the resonator is obtained as follows:

$$
m_{a} \ddot{z}_{a}(t)+c_{a} \dot{z}_{a}(t)+k_{a} z_{a}(t)=-m_{a} \ddot{z}_{l}(t)
$$

According to Kirchhoff's law, the circuit equation of the system is obtained as follows:

$$
\frac{u(t)}{R}+C_{p} \dot{u}(t)=\alpha \dot{z}_{b}(t)
$$

where $C_{p}$ is the equivalent capacitance of the piezoelectric layer. 


\section{2. Continuum modeling and analysis}

The piezoelectric cantilever beam is clamped at one end and free at the other end to form a typical Euler-Bernoulli beam continuum model. The force analysis of the model under excitation is shown in Fig.3. Fig.3 (a) shows the force and motion of the cantilever beam, and $x_{a}$ is the horizontal displacement of the joint between the resonator and the cantilever beam to the fixed end of the cantilever beam. $w_{b}$ is the base displacement, $f_{a}$ is the force of the resonator on the cantilever beam; Fig. 3 (b) is the resonator dynamics model, $z_{a}$ is the displacement of the resonator relative to the cantilever beam, $f_{a}$ is the resonator is cantilever The reaction force of the beam.

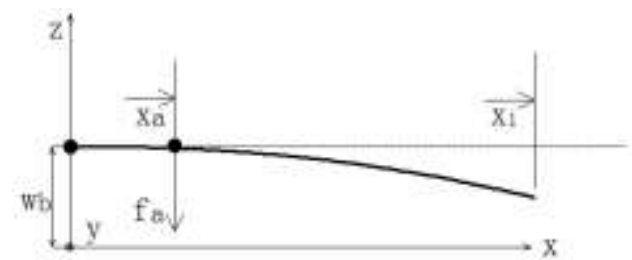

(a). Piezoelectric cantilever motion

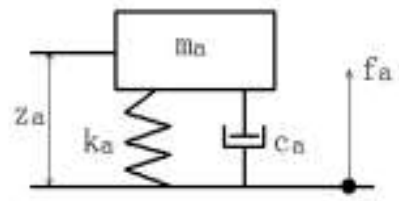

(b). Resonator motion

Fig. 3: Analysis of the force of piezoelectric cantilever beam under excitation.

Introducing the Dirac function, the cantilever beam is written into the form of distributed force by the concentrated force of the resonator [9]:

$$
f_{a}(t)=\delta\left(x-x_{a}\right) f_{a}(t)
$$

The relative displacement $w_{r e l}(x, t)$ of the piezoelectric beam element and the foundation is expressed as the difference between the absolute displacement $w(x, t)$ of the piezoelectric beam element and the foundation displacement $w_{b}(t)$.

$$
w_{\text {rel }}(x, t)=w(x, t)-w_{b}(t)
$$

Due to Fig.3 (a), the motion governing equations of the piezoelectric cantilever beam element in the z-axis direction are as follows according to the Euler-Bernoulli beam theory [10]:

$$
\rho \frac{\partial^{2} w_{r e l}(x, t)}{\partial t^{2}}+\frac{\partial^{2}}{\partial x^{2}}\left[Y I \frac{\partial^{2} w_{r e l}(x, t)}{\partial x^{2}}+c_{S} I \frac{\partial^{3} w_{r e l}(x, t)}{\partial x^{2} \partial t}+\theta u(t)\right]=-\rho \frac{\partial^{2} w_{b}(t)}{\partial t^{2}}+\delta\left(x-x_{a}\right) f_{a}(t)
$$

where $\rho$ is the equivalent density of the piezoelectric beam, $Y I$ is the bending stiffness, $c_{S}$ is the strain coefficient of the strain velocity, $I$ is the moment of inertia of the piezoelectric cantilever beam, $\theta$ is the electromechanical coupling coefficient, $\theta=$ $e_{31} b d_{p} . w_{r e l}(x)$ is the relative displacement of the piezoelectric beam element to the foundation, $u(t)$ is the piezoelectric output voltage. In the second bracket of the left side of the equation, $Y I \frac{\partial^{2} w_{r e l}(x, t)}{\partial x^{2}}+c_{S} I \frac{\partial^{3} w_{r e l}(x, t)}{\partial x^{2} \partial t}+\theta u(t)$ is the bending moment considering the inverse piezoelectric effect [9].

The force $f_{a}$ expression of the resonator on the piezoelectric cantilever beam can be obtained by the mechanical balance principle:

$$
f_{a}=-\left[c_{a} \dot{z}_{a}(t)+k_{a} z_{a}(t)\right]
$$

The equation of motion of the resonator which can be seen from Fig.3 (b) is:

$$
m_{a} \ddot{z}_{a}(t)+c_{a} \dot{z}_{a}(t)+k_{a} z_{a}(t)=m_{a}\left[\ddot{w}_{r e l}\left(x_{a}, t\right)+\ddot{w}_{b}(t)\right]
$$


where $\ddot{w}_{r e l}\left(x_{a}, t\right)+\ddot{w}_{b}(t)$ is the second derivative of the absolute displacement of the cantilever beam element at the resonator. According to Kirchhoff's law, the current in the circuit loop satisfies:

$$
C_{p} \dot{u}(t)+\frac{u(t)}{R}=-\chi_{l} e_{31} z_{p c} b \dot{\eta}_{1}(t)
$$

where $e_{31}$ is the piezoelectric constant, the center coordinate of the $z_{p c}$ piezoelectric piece, and $C_{p}$ is the equivalent capacitance of the piezoelectric layer, which is the same as the lumping model.

The modal superposition algorithm is used to solve the displacement of the piezoelectric cantilever beam:

$$
w_{r e l}(x, t)=\sum_{r=1}^{\infty} \widehat{\emptyset}_{r}(x) \eta_{r}(t)(r=1,2,3 \ldots \ldots)
$$

where $\eta_{r}(t)$ is the modal coordinate; $\widehat{\emptyset}_{r}(x)$ is the mass-normalized mode function; $\beta_{r}$ is the characteristic frequency, which can be obtained by Eq. (11):

where:

$$
\widehat{\phi}_{r}(x)=(\rho L)^{-1 / 2}\left[\cos \left(\beta_{r} x\right)-\cosh \left(\beta_{r} x\right)+\xi_{r}\left(\sin \left(\beta_{r} x\right)-\sinh \left(\beta_{r} x\right)\right)\right.
$$

$$
\xi_{r}=\left[\sin \left(\beta_{r} L\right)-\sinh \left(\beta_{r} L\right)\right] /\left[\cos \left(\beta_{r} L\right)-\cosh \left(\beta_{r} L\right)\right]
$$

The parameter $\beta_{r} L$ can be obtained from the characteristic equation (13):

$$
1+\cos \left(\beta_{r} L\right) \cosh \left(\beta_{r} L\right)=0
$$

The other two modal variables $\chi_{r}$ and $\gamma_{r}$ are respectively:

$$
\begin{aligned}
& \chi_{r}=\left.\frac{d \hat{\phi}_{r}(x)}{d x}\right|_{x=L} \\
& \gamma_{r}=(\rho L)^{-1 / 2} \frac{2 \xi_{1}}{\beta_{r} L}
\end{aligned}
$$

Equation (21) can be obtained by using the orthogonal properties of the cantilever beam:

$$
1+\cos \left(\beta_{r} L\right) \cosh \left(\beta_{r} L\right)=0
$$

where $r$ is the order number. In order to reduce the calculation amount, since the second-order resonance frequency of cantilever beam is far from the first-order resonance frequency, it has a small impact on the first-order mode, and only the first-order mode components are calculated in the time domain integration.

\section{Experimental device introduction}

In order to compare and analyze the accuracy, characteristics and application range of the above two models, the voltage harvesting experiment of the piezoelectric cantilever beam vibration subsystem is carried out. The experimental device is shown in Fig.4. The piezoelectric beam is made of a piezoelectric sheet and a copper substrate, where in the piezoelectric 
sheet is made of PZT-5H piezoelectric ceramic; the additional resonator is formed by a concentrated mass block and a spring. The whole test device is installed on the electromagnetic vibration test bench. The sweep frequency is used to realize the single-step sweep frequency of the system excitation frequency from $9 \mathrm{~Hz}$ to $100 \mathrm{~Hz}$. The output signal is obtained by the data acquisition system by measuring the voltage across the load resistance.

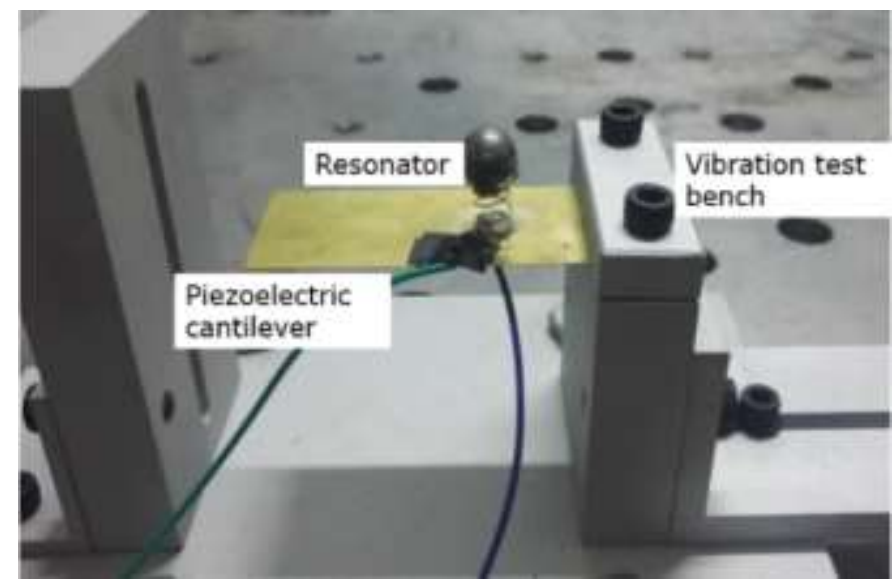

Fig. 4: Piezoelectric cantilever vibration energy harvester experimental device.

In addition, in order to get the change trend of the output voltage with the experimental device parameters, change one of the parameters of the piezoelectric vibration energy harvester device which is the mass of the resonator and the increase the mass of the resonator from $3 \mathrm{~g}$ to $14 \mathrm{~g}$. Then five sets of energy harvest experiments are performed, and data is separately recorded to obtain the change trend of the output voltage.

The material parameters and geometric parameters of the piezoelectric cantilever system are shown in Table 1(a), (b).

Table 1(a): Parameters of piezoelectric cantilever.

\begin{tabular}{ccc}
\hline Parameters & Substrate & Piezoelectric layer \\
\hline Width $\mathrm{b}(\mathrm{mm})$ & 10 & 10 \\
Long $(\mathrm{mm})$ & 60 & 60 \\
Thickness $h(\mathrm{~mm})$ & 0.20 & 0.25 \\
Young's modulus $Y(\mathrm{GPa})$ & 100 & 66 \\
Density $\rho\left(\mathrm{kg} / \mathrm{m}^{3}\right)$ & 8930 & 7500 \\
Piezoelectric constant $d 31\left(10^{-12} \mathrm{C} / \mathrm{N}\right)$ & --- & 275 \\
Capacitance $C_{p}(\mathrm{nF})$ & --- & 37.5 \\
Electromechanical coupling constant $\alpha$ & --- & $6.2 \times 10^{-4}$ \\
Damping ratio $\zeta$ & 0.01 & 0.01 \\
\hline
\end{tabular}

Table 1(b): Other parameters of the whole system.

\begin{tabular}{cc}
\hline Parameters & The numerical \\
\hline Load resistance $\mathrm{R}(\Omega)$ & 1000 \\
Damping ratio $\zeta$ of resonator & 0.0055 \\
Spring stiffness $k_{a}(\mathrm{~N} / \mathrm{m})$ & 613 \\
\hline
\end{tabular}




\section{Comparative analysis of simulation results}

\subsection{The modeling results compared with the experimental data}

Fig. 5 is the characteristic curve of output voltage with frequency change obtained by experimental data, simulation of continuum model and lumping model when the resonator mass increases from $3 \mathrm{~g}$ to $14 \mathrm{~g}$.

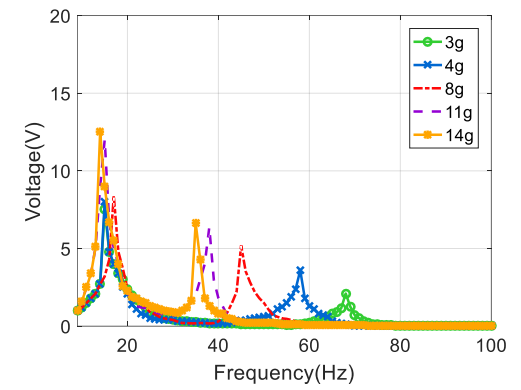

(a) Experimental data

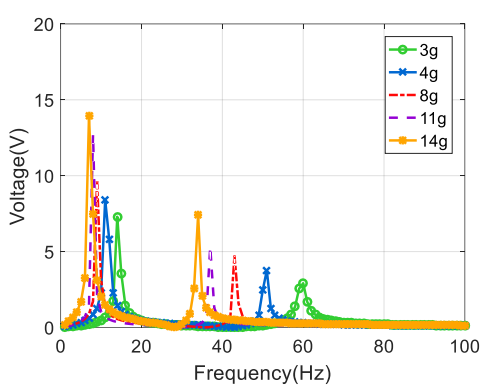

(b) Lumping model

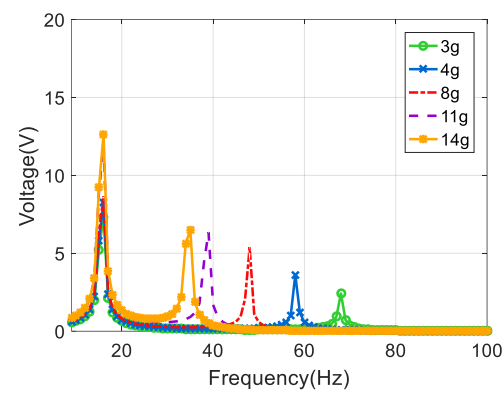

(c) Continuum model

Fig. 5: Comparison of output voltage with changes in resonator mass.

It can be seen from the figure that, whether it is the lumping model or the continuum model, the corresponding change rule at the second-order frequency of the curve is consistent with the experimental data. However, the values of frequency corresponding to the peak and the peak have some deviation.

\subsection{Results analysis and discussion}

According to the modeling process and calculation results of two different mathematical algorithm, the two algorithms are compared and analyzed from four aspects of modeling difficulty, variation trend of peak voltage, peak voltage, peak frequency.

According to the modelling process and calculation results of two different mathematical algorithms, the two algorithms are compared and analysed from four aspects of modelling difficulty, variation trend of peak voltage, peak voltage, and peak frequency.

(1) Trend of peak voltage variation

The overall trend of simulation curves of the two models is consistent with the experimental data: At $0 \sim 50 \mathrm{~Hz}$, the curve presents two peaks. The frequency of the first peak is the natural frequency of the piezoelectric plate, and the frequency of the second peak is the natural frequency of the resonator. After mass changes, experimental data, simulation results of lumping model and continuum model are shown in Fig.5. It can be obtained from the experimental data that the increase of the resonator mass increases the peak voltage corresponding to the two-order natural frequency. The peak voltage obtained by the continuum model and the lumping model increases with the increase of the resonator mass.

(2) Peak voltage

As can be seen from Fig.5, there is a large error between the peak voltage obtained by the lumping model and the experimental data, and error between the peak voltage obtained by most continuum model and the experimental data is less than 5\%. It can be seen from this that the continuum model is more accurate in solving the voltage peak, while the lumping model does not take into account the high-order frequency effect.

(3) The frequency corresponding to the peak

It can be obtained from Fig.5 that: the increase of resonator mass has no effect on the first-order natural frequency, and the second-order frequency will go down. The numerical value and variation trend of the first and second order natural frequencies of the continuum method are in good agreement with the experimental results. The first-order and second-order natural frequencies of the lumping model are generally small, and the first-order natural frequencies tend to decrease with the increase of the resonator mass, but the variation trend of the second order frequency is the same as the experimental value. It can be seen from Fig.5 that the first and second order frequencies obtained by continuum method are very close to 
the experimental data. Because the placement of the resonator will have a great impact on the change of the cantilever beam deformation, the force distribution and the construction deformation of the cantilever beam cannot be ignored when solving the peak frequency, the numerical error between the natural frequency of lumping model and the experimental results is large, especially the natural frequency at the first order.

\section{Conclusions}

By comparing the two modelling algorithm s of the lumping model and the continuum model, and combining the relevant data results of the simulation experiment, the following conclusions can be drawn:

(1) The peak frequency and peak size of lumping model have certain errors, but it is simple and fast, and it can be used for optimization. Solve, adjust the parameters to observe the curve change, predict the peak change, or solve the approximation of the output voltage;

(2) The continuum model considers the deformation and force distribution of the cantilever beam to solve the natural frequency and the output voltage peak, the accuracy is high, but the algorithm is complex. Therefore, to obtain an accurate solution, the continuum model can ensure higher accuracy, and actual more consistent;

(3) In the research of energy harvest, the appropriate model can be selected according to the needs of the research objectives, and the simple model algorithm can be selected as much as possible or combine the two algorithms to achieve time-saving, efficient, and accurate results.

\section{Acknowledgements}

This work is supported by the National Key R \& D Plan of China (Grant No. 2016YFC0802706-01) and University of Science and Technology Beijing.

\section{References}

[1] S. Saadon, O. Sidek, "Vibration-based MEMS piezoelectric energy harvester (VMPEH) modelling and analysis for green energy source," Developments in E-systems engineering, IEEE, 2011, pp. 527-531.

[2] M. Rajarathinam, S. F. Ali, "Energy generation in a hybrid harvester under harmonic excitation," Energy Conversion and Management, vol. 155, pp. 10-19, 2018.

[3] W. Guang-Qing, L. Yue-Ming, "An Improved Lumped Parameter Model for a Piezoelectric Energy Harvester in Transverse Vibration," Shock and Vibration, pp. 1-12, 2014.

[4] S. Rafique, P. Bonello, "Experimental validation of a distributed parameter piezoelectric bimorph cantilever energy harvester," Smart Materials and Structures, vol. 19, no. 9, pp. 71-81, 2010.

[5] S. Leadenham, A. Erturk, "Unified nonlinear electroelastic dynamics of a bimorph piezoelectric cantilever for energy harvesting, sensing, and actuation," Nonlinear Dynamics, vol. 79, no. 3, pp. 1727-1743, 2015.

[6] C. Wei, X. Jing, "A comprehensive review on vibration energy harvesting: Modelling and realization," Renewable and Sustainable Energy Reviews, vol. 74, pp. 1-18, 2017.

[7] N. Jaber, A. Ramini, Q. Hennawi, M. I. Younis, "Wideband MEMS resonator using multi-frequency excitation," Sens Actuat A - Phys, vol. 242, pp. 140-145, 2016.

[8] X. Wang, X. Liang, G. Shu, S. Watkins, "Coupling analysis of linear vibration energy harvesting systems," Mechanical Systems and Signal Processing, vol. 70, pp. 428-444, 2016.

[9] A. Erturk and D. J. Inman, "A distributed parameter electromechanical model for cantilevered piezoelectric energy harvesters," Journal of Vibration and Acoustics-Transactions of the ASME, vol. 130, no. 4, pp. 041002-0410017, 2008.

[10] O. A. Bauchau, J. I. Craig, "Euler-Bernoulli beam theory," Structural analysis, Springer, Dordrecht, 2009, pp. $173-221$. 